\title{
It could be you
}

\section{... a focus for all the staff to work} together in achieving a goal, improving staff motivation and job satisfaction

\begin{abstract}
A $t$ the BDA Conference at Harrogate on 3- 5 May 200 I two innovative and complementary initiatives were Jaunched. One was the BDA's own 'Good Practice Scheme' and the other was the Focus Awards (a joint initiative between the Department of Health and the BDA), and although they have been developed completely separately, they do, in fact, complement each other and both will contribute to standards of dental care for patients.
\end{abstract}

The Good Practice Scheme provides external recognition for those practices and organisations that are committed to the standards of quality care we now accept as appropriate in dentistry. The Focus Awards recognise those practices and organisations that have introduced patient-focused innovations in the last two years. They complement each other beautifully because the Good Practice Scheme is aimed at the practices while the Focus Awards are aimed at the patients, although obviously patients benefit from both.

Although they are complementary, there are some important differences that are worth considering. The Good Practice Scheme applies to the whole UK, whereas the Focus Awards currently only apply to England. The Good Practice Scheme is in an initial phase until October this year, whereas the Focus Awards started with the announcement at the BDA Conference on May 4th and the first awards will be made this September, with further awards in future years (in other words you need to get a nomination in by July 31st for this year's awards). The Good Practice Scheme asks practices to sign up to 10 commitments and put systems into place in order to ensure the practice delivers on those commitments. The Focus Awards asks people to nominate a practice or clinic because of patient-focused innovations in the past two years, either their own practice or another one. The Good Practice Scheme applies to all practices, the Focus Awards is for practices or clinics with at least 50\% NHS commitment (in other words, the vast majority). Finally, while the Good Practice Scheme involves some investment from the practice the Focus Awards offers cash to the winners.

However, the similarities between both initiatives are even more important, because they demonstrate how both enhance each other. Both focus on patients, allowing a practice to use the Good Practice Scheme to help plan for the future while receiving the recognition for a patient-focused approach in the past via the Focus Awards. Both apply to most dental practices and both provide an opportunity for superb promotional activity locally, either by using press releases in the local press or appropriate material internally (such as waiting room posters and patient newsletters). Both will help towards NHS requirements on clinical governance, and both will provide the practice with additional recognition by Health Authorities and other official bodies. Most importantly, both provide a focus for all the staff to work together in achieving a goal, ias well as improving staff motivation and job satisfaction.

It would be too easy for both initiatives to be dismissed as 'yet more official interference' or 'just another way of checking up on us'; but that is surely missing the point. As I have said in previous leaders, initiatives like CPD or Investors in People are there to be taken advantage of. Both these current initiatives offer tremendous opportunities to people with vision, because they open doors without prescribing activity. You do not have to do specific things to achieve either (or both). You simply need to see how best you can fit existing patient care into the formats that exist for both initiatives (with appropriate changes if that helps) and apply. As all the activities that make up both initiatives help build better, more caring and probably more profitable practices, everyone benefits. 